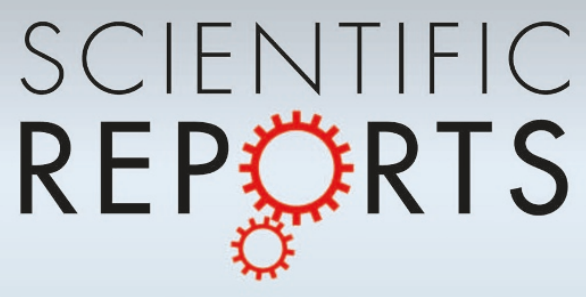

OPEN

SUBJECT AREAS:

BIOSENSORS

SPECTROPHOTOMETRY

NANOPARTICLES

CELL DEATH AND IMMUNE RESPONSE

Received

7 April 2014

Accepted

9 July 2014

Published

26 August 2014

Correspondence and requests for materials should be addressed to Q.M.Q. lquan@ rowland.harvard.edu)

\section{Nanoscale Label-free Bioprobes to Detect Intracellular Proteins in Single Living Cells}

\author{
Wooyoung Hong ${ }^{1,2}$, Feng Liang ${ }^{2}$, Diane Schaak ${ }^{2}$, Marko Loncar ${ }^{3} \&$ Qimin Quan ${ }^{2}$
}

\begin{abstract}
'Department of Chemistry and Chemical Biology, Harvard University, Cambridge, MA 02138, USA, ${ }^{2}$ Rowland Institute at Harvard University, Cambridge, MA 02142, USA, ${ }^{3}$ School of Engineering and Applied Sciences, Harvard University, Cambridge, MA 02138 , USA.
\end{abstract}

\begin{abstract}
Fluorescent labeling techniques have been widely used in live cell studies; however, the labeling processes can be laborious and challenging for use in non-transfectable cells, and labels can interfere with protein functions. While label-free biosensors have been realized by nanofabrication, a method to track intracellular protein dynamics in real-time, in situ and in living cells has not been found. Here we present the first demonstration of label-free detection of intracellular p53 protein dynamics through a nanoscale surface plasmon-polariton fiber-tip-probe (FTP).
\end{abstract} abel-free technology is ideal for in vivo and in situ detection in primary cells, tissue, or living systems. To date,
intracellular action potential has been measured with three-dimensional nanowire field-effect transistors ${ }^{1}$, - intracellular $\mathrm{pH}$ has been measured with nanofibers ${ }^{2}$, and cellular endoscopy has been illustrated with carbon nanotubes ${ }^{3}$, nanowires ${ }^{4}$, and nanobeams ${ }^{5}$. Vo-Dinh et. al. further demonstrated the detection of enzymatic fluorescent intracellular proteins by integrating immunoassay with nanofibers ${ }^{6,7}$. However, label-free tracking of protein concentrations in live cells repeatedly over time has yet to be developed. Here we describe a localized surface plasmon resonance (LSPR) fiber tip probe (FTP) system and demonstrate label-free detection of intracellular p53 tumor suppressor protein, which has been widely regarded as a key target for cancer therapy ${ }^{8,9}$. We demonstrate our technology by detecting the concentration of p53 in single HeLa cells in response to ultraviolet radiation and treatment with neocarzinostatin (NCS). Our method is widely applicable to other intracellular proteins, as well as having the potential to determine when, where, and under what conditions specific proteins are created and disintegrated.

The FTP is a tapered optical fiber that has a sub-5 micrometer length and sub-100 nanometer diameter tip (Fig. 1a and b). A single gold nanorod (Nanopartz Inc.) was attached to the end of the tip, the surface of which was functionalized with antibodies specific to the target analyte proteins. The collective oscillation of the conductive electrons in the gold nanorod couples strongly to light and generates a resonant scattering signal (LSPR) ${ }^{10}$. The light, strongly localized on the surface of the gold nanorod, is sensitive to small perturbations of its optical mode near its surface ${ }^{11-13}$. Therefore, by monitoring the resonance shift of the LSPR, one can quantify the binding of analyte proteins to the sensor surface. Due to the nanoscale tip size, the FTP entails minimum invasiveness when inserted into the cell membrane.

\section{Results}

A scanning electron microscope (SEM) image of the FTP is shown in Fig. 1a. A zoomed image of its nanosize tip attached with a single gold nanorod is shown in Fig. 1b. A white light source (halogen lamp) was connected to the fiber and excited the LSPR of the gold nanorod at the tip. The resonant scattering signal (red glowing in Fig. 1c) from the gold nanorod on the tip was visible to the naked eye. The resonant scattering signal was collected via $50 \times$ objective and was coupled to a spectrometer (Princeton Instrument). Fig. 1d shows the image on the spectrometer CCD when FTP was immersed in 10 mM Phosphate Buffer Saline (PBS), pH 7.4. Spectrum profile analysis was performed by binning a few pixels around the bright spot to increase the signal-to-noise ratio. By fitting resonance spectrum with the Lorentzian profile, we found that the LSPR resonance of this gold nanorod was at $729 \mathrm{~nm}$, with quality factor of $\sim 10$.

To test the sensitivity of our LSPR-FTP, we characterized it in an in vitro aqueous environment. First, we used FTP to detect different concentrations of glycerol in water. We prepared solutions with $20 \%, 40 \%, 60 \%$, and $80 \%$ of glycerol, and injected them through a microfluidic channel. The FTP was inserted into this channel and sealed with epoxy. The LSPR signal, excited through the fiber coupled with halogen light source, was monitored with 

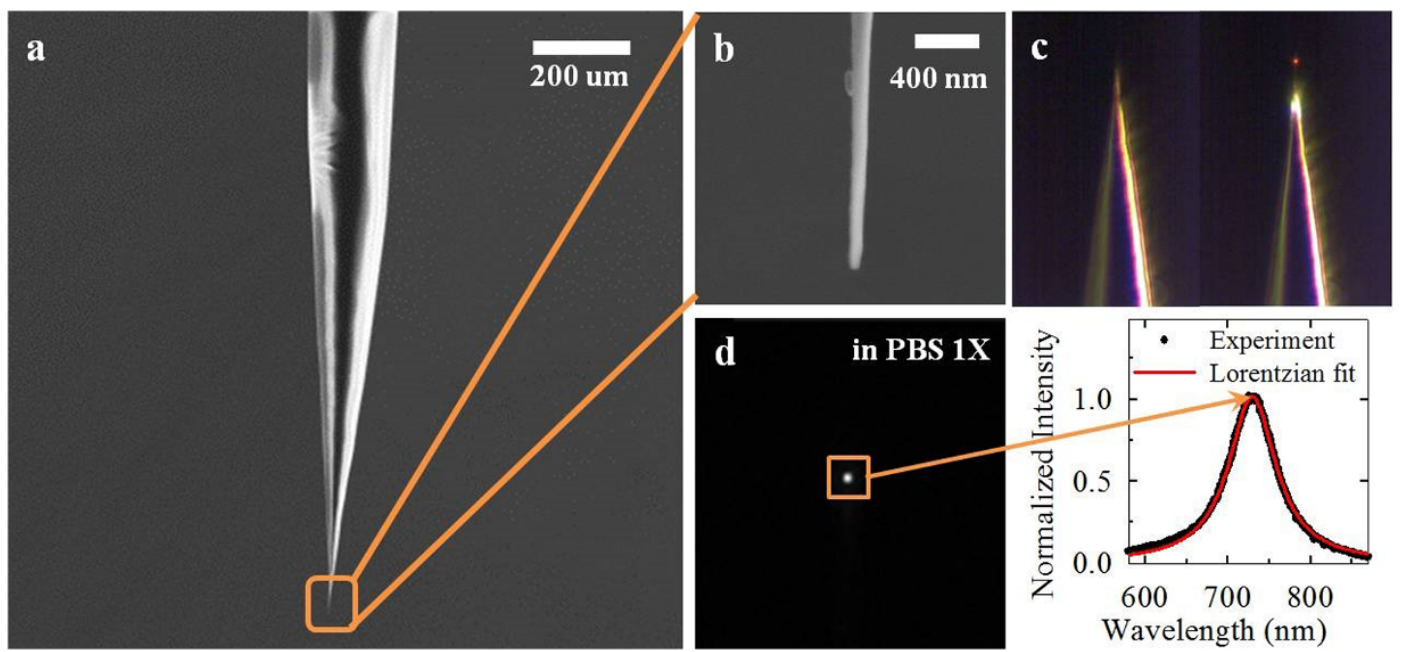

Figure 1 Gold nanorod Fiber tip probe (FTP). (a), Scanning Electron Microscope (SEM) image of a FTP. The optical fiber is gradually tapered from $125 \mathrm{um}$ to sub-100 nm. (b), Enlarged SEM image of the end of a FTP showing a single nanorod immobilized on the FTP. (c), Optical image of the LSPR FTP with excitation light source off (left) and on (right) in air. A red dot (right) corresponds to the LSPR of a gold nanorod. In this experiment, white-light from Halogen source was coupled into the fiber and used to excite the gold nanorod at the fiber tip. (d), Image from the spectrometer CCD at the zeroth order grating (left) and a LSPR spectrum at the first order grating (right). This FTP was immersed in $10 \mathrm{mM}$ phosphate buffered saline (PBS).

inverted microscope (Olympus IX71) (Fig. 2a). Fig. 2b shows representative spectra for each concentration. Fig. $2 \mathrm{c}$ shows the realtime measurement of the LSPR peak wavelength (from Lorentzian fitting). Fig. $2 \mathrm{~d}$ shows the resonance wavelength vs. refractive index of the solution. The LSPR shifted to longer wavelength in the presence of higher concentration of glycerol due to the increase in the refractive index of the solution ${ }^{14}$. Bulk index sensitivity of the FTP is defined as the resonance shift per unit refractive index change in the surrounding medium. We found that the sensitivity of the current device was $187 \pm 25 \mathrm{~nm} / \mathrm{RIU}$ (repeated for five times), signifi- a

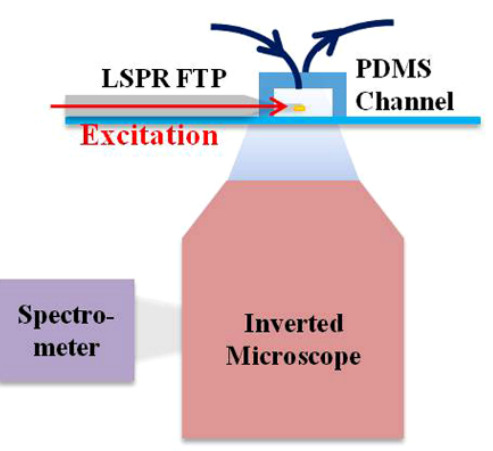

c

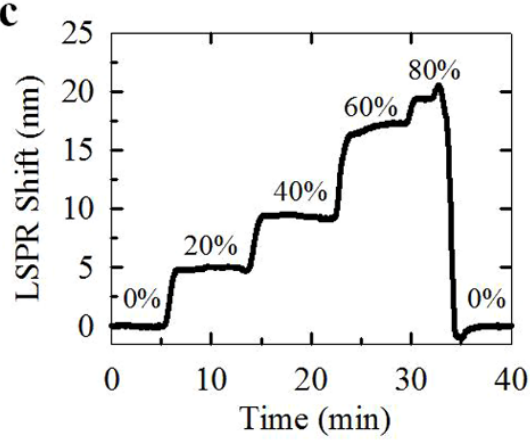

b

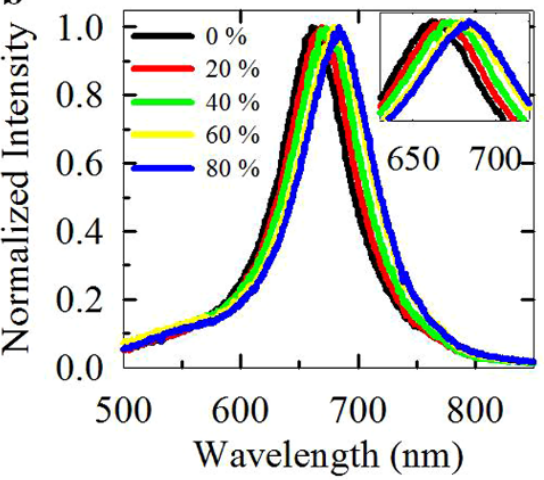

d

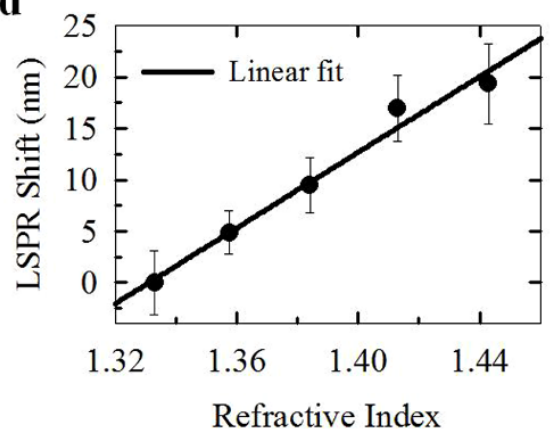

Figure $2 \mid$ In-vitro characterization of the LSPR FTP. (a), Schematic setup for in-vitro characterization of the LSPR FTP. The FTP sensor was assembled into a home-made PDMS channel. The LSPR signal excited through the fiber was collected via an inverted microscope and analyzed by a spectrometer. (b), Normalized LSPR signal in various glycerol/water concentrations. Inset shows the zoomed-in view of LSPR peaks (c), Real-time monitoring of the LSPR when different concentrations of solutions are injected in the channel. The resonance wavelength is obtained by fitting LSPR signals to the Lorentzian equation. (d), Dependence of the LSPR position on the refractive indices of the solvents. The error bar is from the measurements of five different nanorods. A bulk index sensitivity of $187 \pm 25 \mathrm{~nm} / \mathrm{RIU}$ is obtained from linear fit. 

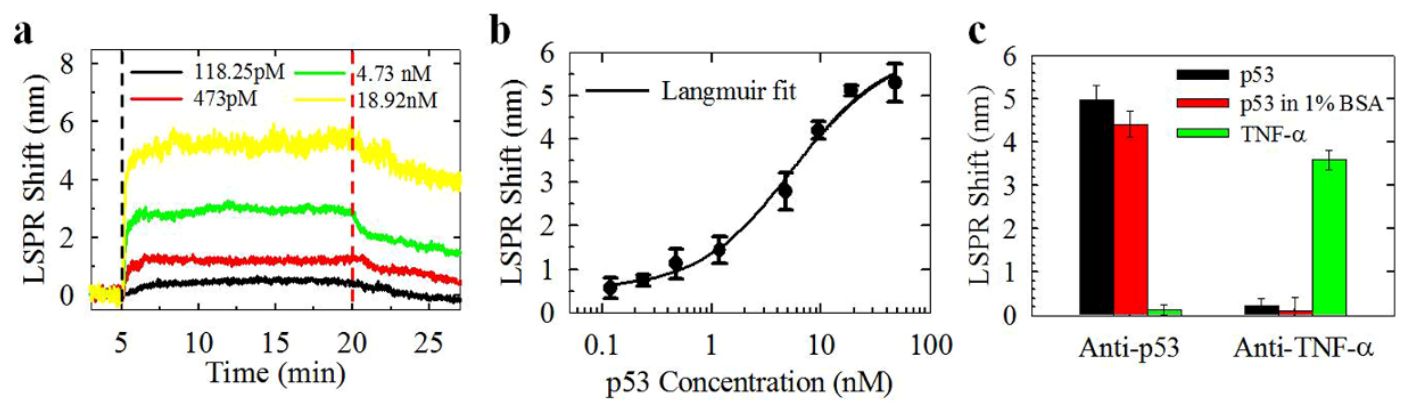

Figure $3 \mid$ In vitro characterization of the LSPR FTP in physiology condition. (a), Real-time measurement of LSPR shifts at various p53 concentrations. In all cases, the blank PBS was injected into the fluidic channel for the first 5 minutes (before black dashed line). Different concentrations of p53 in PBS were injected into the channel for 15 minutes, followed by additional PBS wash step to observe dissociation of p53 from its antibody (after red dashed line). (b), LSPR shift vs. p53 concentration. Dissociation constant of $5.4 \mathrm{nM}$ is extracted from the fit with Langmuir equation. Three repeated experiments were performed. (c), Specificity test of LSPR FTP sensor. The LSPR FTPs were coated with anti-p53 and anti-TNF- $\alpha$ respectively, and both sensors were used to detect p53, p53 in 1\% BSA, and TNF- $\alpha$. The significant LSPR shift occurred when coated antibodies paired correctly with their antigens, thus demonstrating high sensor specificity. High specificity retains when the p53 antigens are in high background of BSA.

cantly greater than that of the $39 \mathrm{~nm}$ diameter gold spheres $(80 \mathrm{~nm} /$ RIU ${ }^{15}$. Gold nanorods are also advantageous over gold spheres because of their reduced damping loss (thus higher Q-factor) ${ }^{16}$.

Second, we used the FTP to detect different concentrations of proteins in the PBS solution. The gold nanorod on the tip of the FTP was functionalized with p53 antibody (Santa Cruz sc-126). Different concentrations of p53 (Santa Cruz sc-4246) in PBS were injected into the fluidic channel for 15 minutes, followed by washing with pure PBS. Fig. 3a shows the real-time response at several representative concentrations. LSPR shift of $5.13 \mathrm{~nm}$ was observed upon introducing $18.92 \mathrm{nM} \mathrm{p} 53$ solution. After washing with pure PBS at the 20th minute, the resonance decreased slowly, exhibiting the dissociation kinetics. A LSPR shift of $0.55 \mathrm{~nm}$ was observed from $1 \mathrm{nM}$ p53 solution, close to the detection limit of this system. The minimum discernible wavelength shift was $\sim 0.3 \mathrm{~nm}$, estimated from the LSPR response to pure PBS flowed for the first five minutes (black dashed line in Fig. 3a). Fig. 3b shows the Langmuir fitting of LSPR shifts at different concentrations. The dissociation constant of p53 from anti-p53 is $5.4 \mathrm{nM}$, in good agreement with published results obtained using commercial instrument ${ }^{17}$.

To investigate the specificity of the system, we functionalized the FTPs with antibodies of either tumor suppressor p 53 or tumor necrosis factor (TNF- $\alpha$ ) and used these FTPs to detect p53 and TNF- $\alpha$ (Fig. 3c). When the FTP was functionalized with anti-p53, it could detect p53 in both pure PBS and PBS with concentrated Bovine Serum Albumin (1\% BSA) as background. On the other hand, the sensor was not sensitive to TNF- $\alpha$, a mismatched antigen-antibody pair. The FTP functionalized with anti-TNF- $\alpha$ also showed high specificity to TNF- $\alpha$ only (Fig. $3 c$ ). This demonstrates that the LSPR FTP shows high specificity to the targeted proteins. Because LSPR of the nanorod has exceptionally smaller optical mode volume $^{18}$ (on the order of the physical dimension of the nanorod) compared to other optical resonators ${ }^{19}$, the sensor is sensitive only within a few nanometers from its surface and is insensitive to untargeted proteins $s^{20}$.

\section{Discussion}

The FTP offers a unique three-dimensional nanoscale form-factor and minimum invasion in cells; thus, it is an ideal intracellular bioprobe for single cell diagnostics. Here we demonstrate how p53 in HeLa cells dynamically respond to ultra-violet (UV) exposure and neocarzinostatin (NCS) drug treatment. We first validated the commercial anti-p 53 to intracellular $\mathrm{p} 53$ by performing western blot with HeLa cell lysis (see supplementary information). In order to minimize the invasiveness to the cell, anti-p53 functionalized FTP was inserted in and retracted out of the cell every 30 minutes. During each measurement, we first inserted the FTP into the cytoplasm of the cell, incubated for 5 minutes, retrieved the FTP out of the cell, and measured its LSPR in the cell culture medium adjacent to the cell of interest. This process was conducted to minimize the scattering background from the cytoplasm when FTP was inside the cell, as well as to wash off the non-specific bindings of other proteins inside the cell. LSPR measurement was immediately taken after the retraction to prevent the unbinding of $\mathrm{p} 53$ from anti-p53. After the FTP was inserted into the cell, we were able to clearly observe a single gold nanorod glowing inside the cell and gradually dimming down after a few minutes (Fig. 4b). This dimming behavior is attributed to the scattering background from the possible non-specific binding of cytoplasmic proteins onto the nanosize tip, evidencing the insertion of the FTP into the cytoplasm.

Before performing the aforementioned measurement, the intensity of UV light source was first calibrated. Live HeLa cells were exposed by different dosages of UV light or different concentrations of NCS. The FTP experiments were performed immediately after the treatments. The results of our experiment indicate that different stresses triggered different temporal responses of p53. As shown in Fig. 4c, we quantified the dynamics of p53 in individual cells in response to different UV exposure and observed an increase in amplitude when the UV dose increased. This dose-dependent escalation of p53 in relation to UV is a clear contrast to the repeated pulses in response to NCS. As is indicated by Fig. 3a, p53 slowly dissociates off the sensor when the background p53 concentration is decreased, and full dissociation requires relatively long time (see supplemental information). The excess un-dissociated p53 adds to residue LSPR shifts from the sensor, and FTP may give higher LSPR shifts than the actual intracellular p53 concentration when p53 is down regulated. This problem can be remedied by regenerating the sensor or by using alternative affinity probes, such as aptamer or cellular receptors.

As a control experiment, we performed the same procedure on an intact HeLa cell. The tumor suppressor protein p53 activity is not required during normal cell growth and exists at only low levels predominantly in the nucleus and lesser in the cytoplasm ${ }^{21,22}$. Fig. $4 \mathrm{c}$ indicates that the intracellular p53 level remained constant at low level. In another control experiment (Fig. 4d), to confirm that the HeLa cell was alive even after a series of measurements ( 8 hours long), a fluorometric assay of calcein was used (Invitrogen LIVE/ DEAD). Our results agree with the previous studies of p53 using fluorescent microscopy and western blot ${ }^{23-25}$.

The FTP technology offers a range of advantages. First, it is a labelfree technology with no fluorescent reporters, which also precludes the interference of labels with proteins. Second, it is capable of interrogating single cells alive, without the need of cell harvest and cell 

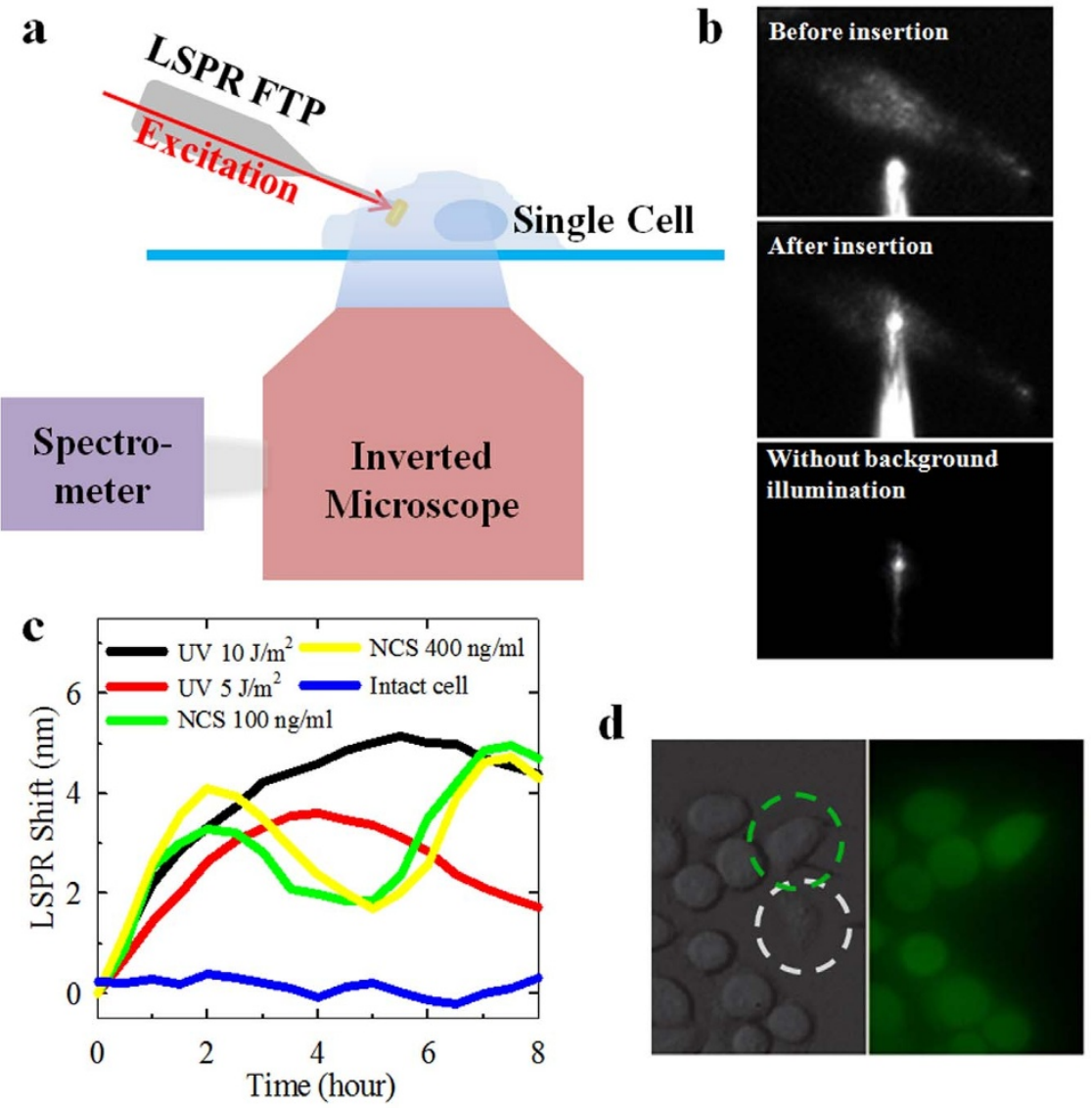

Figure $4 \mid$ Intracellular p53 detection in a single living HeLa cell with FTP. (a), Schematic set-up of the FTP system. The probe, consisting of a nanorod immobilized on the sub-100 $\mathrm{nm}$ tapered tip of an optical fiber, is inserted into a single living cell at designated positions using a three-axis micromanipulator. (b), Sequence of EMCCD images of a FTP penetrating a single HeLa cell, viewed from below. The FTP was first positioned outside the cell with its tip lying slightly flat against the petri dish substrate. It is then punctured into the side of the cell. Without background illumination, we could clearly see the LSPR signal from the nanorod glowing inside the cell. (c), p53 dynamics in the intact, UV exposed and NCS treated HeLa cells. The p53 showed sustained concentration increase under UV, and pulsed oscillation under NCS. (d), Phase-contrast and fluorescent image of cells that have been punctured by FTP. A cell in the green circle fluoresced in green after treatment with calcein viability dye and thus was alive after a series of measurement ( 8 hours long) with nanosize FTP. The cell showed similar levels of green fluorescence intensity indicating the viability of both poked and intact cells. However, a cell in the white circle was dead after being punctured once by a FTP with 5-micrometer tip in diameter.

lysis. Third, it allows the quantification of the real-time, intracellular dynamics. By referring to the measurement in in-vitro environment, we estimate that the $\mathrm{p} 53$ concentration can increase up to the order of $10 \mathrm{nM}$ under external stress.

Combining the advantages of high selectivity from antibody-antigen recognition and high sensitivity of LSPR, we demonstrated a novel three-dimensional nanoscale bioprobe for intracellular protein detection. Our method is an alternative approach to fluorescent labeling approach, and will be especially useful for cells and systems that are hard to label, such as immune cells, neurons, and precious patients' cells and in a wide array of circumstances that require in vivo and in situ detection. The ability to manipulate LSPR FTP three dimensionally ensures the minimal and local invasion of the probe into the cell and high reproducibility of the results. While current demonstration regards a single bioprobe detecting one specific protein in single cells, our method has the potential to extend to a highthroughput multiplexed system, using fiber arrays or nanowire arrays.

\section{Methods}

Device fabrication. The FTP was fabricated by wet-etching an optical fiber (SM28, Thorlabs Inc.) with hydrogen fluoride chemistry by precisely monitoring and controlling the etch time (details see supplementary information). Gold nanorods (Nanopartz Inc.) were dispersed on a cover slip and then picked up onto the nanosize tip of the FTP by using 3D micro-manipulator under the dark field of an inverted microscope (details see supplementary information). UV-curing optical adhesive (Norland NOA 138) was used to adhere a nanorod to the tip.

Surface functionalization. Details of the surface functionalization procedure can be found in the supplementary information. In brief summary, $1 \mathrm{~mL}$ of cetrimonium bromide (CTAB)-capped gold nanorods (Nanopartz Inc.) was mixed with $100 \mu \mathrm{L}$ of a $20 \mathrm{mM}$ solution of 11-mercaptoundecanoic acid (11-MUA, Sigma-Aldrich) prepared in ethanol. The mixture was sonicated for 90 minutes at $55^{\circ} \mathrm{C}$ and kept at room temperature overnight. These nanorods were centrifuged at $7000 \mathrm{rpm}$ for 10 minutes and redispersed in $\mathrm{H}_{2} \mathrm{O}$ to remove the excess MUA. One of these nanorods was immobilized on the tip of the FTP mounted on the micromanipulator. The assembled LSPR FTP was incubated in $100 \mathrm{mM}$ 1-Ethyl-3-(3-

dimethylaminopropyl)carbodiimide (EDC, Sigma-Aldrich) and $100 \mathrm{nM}$ anti-p53 (Santa Cruz) in $10 \mathrm{mM}$ PBS. EDC molecules react to the carboxyl group of MUA molecules, which caps the gold nanorod, and form unstable esters, which react to the amine groups on the antibodies. Anti-p53 coated on the nanorod is then prepared to specifically capture p53 proteins.

LSPR FTP characterization. Information regarding the polarization-dependent characterization of the LSPR from nanorod is included in the supplementary information. LSPR from single nanorod was also compared with the UV-Vis spectrum from ensemble nanorods, showing narrower spectrum line-width.

Cell culture, immunoblot and viability test. Human cervix epithelial HeLa cells (American Type Culture Collection, ATCC) were cultured at $37^{\circ} \mathrm{C}$ in DMEM F-12 Medium supplemented with $10 \%$ fetal calf serum, $100 \mathrm{U} / \mathrm{mL}$ penicillin, and $100 \mu \mathrm{g} / \mathrm{mL}$ streptomycin. Western blot (see supplementary information) was conducted to validate the affinity of the anti-p53 (Santa Cruz) to the commercial p53 protein (Santa Cruz) as well as to the p53 protein from the HeLa cell lysis. Upon completion of the experiment, the subject cells were tested with the calcein AM cell viability assay (Invitrogen). 
1. Tian, B. et al. Three-Dimensional, Flexible Nanoscale Field-Effect Transistors as Localized Bioprobes. Science 329, 830-834 (2010).

2. Tan, W., Shi, Z. Y., Smith, S., Birnbaum, D. \& Kopelman, R. Submicrometer intracellular chemical optical fiber sensors. Science 258, 778-781 (1992).

3. Singhal, R. et al. Multifunctional carbon-nanotube cellular endoscopes. Nat Nanotechnol. 6, 57-64 (2011).

4. Yan, R. et al. Nanowire-based single-cell endoscopy. Nat. Nanotechnol. 7, 191-196 (2012).

5. Shambat, G. et al. Single-cell photonic nanocavity probes. Nano Lett. 13, 4999-500 (2013).

6. Vo-Dinh, T., Alarie, J. P., Cullum, B. M. \& Griffin, G. D. Antibody-based nanoprobe for measurement of a fluorescent analyte in a single cell. Nat. Biotechnol. 18, 764-767 (2000).

7. Vo-Dinh, T. \& Kasili, P. Fiber-optic nanosensors for single-cell monitoring. Anal. Bioanal. Chem. 382, 918-925 (2005).

8. Levine, A. J. \& Oren, M. The first 30 years of p53: growing ever more complex. Nat. Rev. Cancer 9, 749-758 (2009).

9. Lane, D. P., Cheok, C. F. \& Lain, S. p53-based cancer therapy. Cold Spring Harb. Perspect. Biol. 2, a001222 (2010).

10. Anker, J. N. et al. Biosensing with plasmonic nanosensors. Nat. Materials 7, 442-453 (2008).

11. Kall, M. Biosensors: One molecule at a time. Nat. Nanotechnol. 7, 347-349 (2012).

12. Ament, I., Prasad, J., Henkel, A., Schmachtel, S. \& Sonnichsen, C. Single unlabeled protein detection on individual plasmonic nanoparticles. Nano Lett. 12, 1092-1095 (2012).

13. Nusz, G. J. et al. Label-free plasmonic detection of biomolecular binding by a single gold nanorod. Anal. Chem. 80, 984-989 (2008).

14. Chen, H. et al. Shape-Dependent Refractive Index Sensitivities of Gold Nanocrystals with the Same Plasmon Resonance Wavelength. J. Phys. Chem. C 113, 17691-17697 (2009).

15. Nath, N. \& Chilkoti, A. Label-free biosensing by surface plasmon resonance of nanoparticles on glass: optimization of nanoparticle size. Anal. Chem. 76, 5370-5378 (2004).

16. Sonnichsen, C. et al. Drastic reduction of plasmon damping in gold nanorods. Phys. Rev. Lett. 88, 077402 (2002).

17. Kastelic, D. et al. A single-step procedure of recombinant library construction for the selection of efficiently produced llama $\mathrm{VH}$ binders directed against cancer markers. J. Immunol. Methods 350, 54-62 (2009).

18. Brolo, A. G. Plasmonics for future biosensors. Nat. Photonics 6, 709-713 (2012).

19. Vahala, K. J. Optical microcavities. Nature 424, 839-846 (2003).

20. Yonzon, C. R. et al. A comparative analysis of localized and propagating surface plasmon resonance sensors: the binding of concanavalin a to a monosaccharide functionalized self-assembled monolayer. J. Am. Chem. Soc. 126, 12669-12676 (2004).

21. O’Keefe, K., Li, H. P. \& Zhang, Y. P. Nucleocytoplasmic shuttling of p53 is essential for MDM2-mediated cytoplasmic degradation but not ubiquitination. Mol. Cell. Biol. 23, 6396-6405 (2003).

22. Green, D. R. \& Kroemer, G. Cytoplasmic functions of the tumour suppressor p53. Nature 458, 1127-1130 (2009).

23. Purvis, J. E. et al. p53 dynamics control cell fate. Science 336, 1440-1444 (2012)

24. Khosravi, R. et al. Rapid ATM-dependent phosphorylation of MDM2 precedes p53 accumulation in response to DNA damage. Proc. Natl. Acad. Sci. USA 96, 14973-14977 (1999).

25. Batchelor, E., Loewer, A., Mock, C. \& Lahav, G. Stimulus-dependent dynamics of p53 in single cells. Mol. Syst. Bio. 7, 488 (2011).

\section{Acknowledgments}

Q.Q. acknowledges the support of this work by Rowland Institute at Harvard University. W.H. acknowledges support from the Samsung fellowship.

\section{Author contributions}

W.H. and Q.Q. conceived of the experiment, W.H. performed the experiments, F.L. and D.S. cultured the cell, W.H. and Q.Q. analyzed the data and wrote the paper. W.H., Q.Q. and M.L. discussed the results and commented on the manuscript.

\section{Additional information}

Supplementary information accompanies this paper at http://www.nature.com/ scientificreports

Competing financial interests: The authors declare no competing financial interests.

How to cite this article: Hong, W., Liang, F., Schaak, D., Loncar, M. \& Quan, Q. Nanoscale Label-free Bioprobes to Detect Intracellular Proteins in Single Living Cells. Sci. Rep. 4, 6179; DOI:10.1038/srep06179 (2014).

(i) $\Theta$ This work is licensed under a Creative Commons Attribution-NonCommercialNoDerivs 4.0 International License. The images or other third party material in this article are included in the article's Creative Commons license, unless indicated otherwise in the credit line; if the material is not included under the Creative Commons license, users will need to obtain permission from the license holder in order to reproduce the material. To view a copy of this license, visit http:// creativecommons.org/licenses/by-nc-nd/4.0/ 